\title{
Fully Covered Self-Expandable Metal Stent in Tracheobronchial Disorders: Clinical Experience
}

\author{
Roberto Marchese $^{a} \quad$ Grazia Poidomani $^{a} \quad$ Giuseppe Paglino ${ }^{a} \quad$ Claudia Crimic $^{c}$ \\ Chiara Lo Nigro ${ }^{a}$ Vincenzo Argano ${ }^{b}$ \\ a Interventional Pulmonology Unit, La Maddalena Cancer Center, and b Cardiac Surgery Unit, Azienda Ospedaliera \\ Policlinico, Palermo, and 'Intensive Respiratory Care Unit, Azienda Ospedaliera Cannizzaro, Catania, Italy
}

\section{Key Words}

Airway obstruction · Fistula · Fully covered self-expandable metallic stent

\begin{abstract}
Background: The third-generation fully covered self-expandable metallic stent (SEMS) has been developed to solve the problems of difficult removal and in-stent granuloma formation related to the uncovered or partially covered type. There are few written reports about the performance of this type of stents with early encouraging results. Objectives: To report and analyse our experience with the Silmet ${ }^{\circledR}$ stent in the management of malignant and benign tracheobronchial disorders. Methods: We retrospectively reviewed medical records of patients who underwent fully covered SEMS Silmet placement at the Interventional Pulmonology Unit, La Maddalena Cancer Center, Palermo, Italy, between May 2010 and August 2013. Results: Stents were placed in 52 patients with malignant $(n=49)$ and benign airway obstruction $(n=$ $2)$ and broncho-oesophageal fistula $(n=1)$. SEMSs were inserted into the trachea $(n=19)$, the main bronchi $(n=21)$ and the peripheral bronchi $(n=31)$. Besides 1 procedural dislocation, the deployment was successful in all patients with an
\end{abstract}

(c) 2015 S. Karger AG, Basel

0025-7931/15/0891-0049\$39.50/0 immediate significant improvement of symptoms (Barthel Index $p<0.001$; Medical Research Council score $p<0.001$ ). A radiographic improvement was detected in $48 \%$ of patients. The mean follow-up duration was $119 \pm 120$ days (range 22-549 days). Complications observed were: migration $(7.6 \%)$, tumour overgrowth (15\%), infections $(5.7 \%)$, granulation tissue formation (3.8\%) and mucus plug (3.8\%). Conclusions: The Silmet stent is effective, safe and simple to implant and remove. We suggest its use in cases of tight stenoses, in the treatment of small- to medium-caliber airways or in cases of tortuous airways.

(c) 2015 S. Karger AG, Basel

Airway stenting has become a strengthened bronchoscopic procedure for the treatment of tracheobronchial disorders that has been used for almost 90 years [1]. Several studies have proved the effectiveness of stents as both palliative and curative treatment of lung cancer; in fact they lead to a significant improvement not only in symptoms and lung function [2-11], but also in quality of life [12] and survival, if implanted in time $[13,14]$.

Various models of stents with several different types of materials have been developed, but basically they can be

\section{KARGER 125}

E-Mail karger@karger.com www.karger.com/res
Dr. Roberto Marchese

Interventional Pneumology Unit, La Maddalena Cancer Center

Via San Lorenzo Colli 312

IT-90100 Palermo (Italy)

E-Mail momarc313@yahoo.it 
divided into two groups: polymer and metal stents. The first need to be placed by rigid bronchoscopy but have several advantages such as ease of removal, low rate of granuloma tissue formation, no tumour ingrowth and low cost; the downsize of polymer stents is that they are stiff and have an unfavourable relationship between the internal and external diameters; therefore they have a poor adaptability to a varying airway such as in cases of tight and tortuous stenosis. Moreover, they have the major disadvantage of a high migration rate. Metal stents (SEMS) are made from metal alloys (such as nickel, titanium or nitinol), which are self-expanding [15]; therefore they can be successfully implanted with a flexible bronchoscope even under conscious sedation and local anaesthesia [16]. Unlike silicone stents, SEMS have several advantages such as a lower migration rate, a greater crosssectional airway diameter owing to thinner wall construction, a better conformation to irregular airways, a good epithelialization within the stent that allows for mucociliary clearance and a greater ease of placement. SEMS showed a relatively low complication rate, mainly caused by tumour ingrowths and granulation formation at the stent extremities, stent fracture or perforation and the main disadvantages of being pretty difficult to remove and fairly expensive $[17,18]$.

Third-generation fully covered SEMS such as Silmet ${ }^{\circledR}$ have been developed to solve the problems of difficult removal and/or in-stent granuloma formation [17, 19-23].

Although stent placement is becoming a very popular therapeutic option for patients with tracheobronchial disorders, few written reports about Silmet performance are available in the scientific literature, with early encouraging results $[24,25]$.

We report our experience with SEMS using Silmet in patients with symptomatic malignant and benign tracheobronchial disorders.

\section{Materials and Methods}

We retrospectively reviewed medical records of patients who underwent fully covered SEMS Silmet (Novathec, La Ciotat, France) placement at the Interventional Pulmonology Unit, La Maddalena Cancer Center, Palermo, Italy, between May 2010 and August 2013.

Silmet is a self-expanding stent made of a specific nitinol wire mesh of $5 \mathrm{~mm}$ in length coated with silicone. It has radio-opaque gold markers and is fully covered by polyester. This is commercially available as straight and conical stent in various diameters and lengths.

Indications for tracheobronchial stent implantation were symptomatic benign and neoplastic tracheobronchial disorders.
Early and late outcomes were identified from the patient's medical notes. Data gathered included patient demographics, clinical presentation, indication for stent, location and size of stent and any complication identified.

All the patients were nonsurgical candidates, and stent placement was judged to be the only therapeutic option for these symptomatic patients.

Informed consent was obtained from all patients before bronchoscopic SEMS implantation and follow-up.

Before the airway stent placement, each patient underwent clinical assessment and broncoscopy (model 1T-180; Olympus America Inc., Melville, N.Y., USA) under local anaesthesia to evaluate airway anatomy and to plan the best approach for treatment. All stents were placed by rigid bronchoscopy (Dumon-Harrell type; Bryan Corp., Woburn, Mass., USA) under general anaesthesia and jet ventilation.

A straight or conical SEMS Silmet was implanted under direct vision only when support and tutorage of the airways was necessary. Laser $(\lambda=980 \mathrm{~nm}$; Ceralas D50/980/600; Biolitech, Bonn, Germany) therapy was used in case of endoluminal lesions.

After the procedure the patients were moved to a high dependency unit for 1-2 $\mathrm{h}$ for observation and then transferred to an ordinary ward area.

Chest X-ray was usually performed the day before and after the procedure to check for complication and to highlight a radiographic improvement in terms of resolution of atelectasis/infiltrates or presence of mediastinal shift.

According to the protocol of our department, the patient's follow-up programme consisted of 4 complete visits (V1 at $24 \mathrm{~h}$ after stent placement; V2 at 1 month after the procedure; V3 at 3 months after the procedure; V4 at 6 months after the procedure) during which pulmonary function, Barthel Index [26] and Medical Research Council (MRC) dyspnoea scale were measured to assess all patients' clinical status and improvement. During every visit, flexible bronchoscopy under local anaesthesia was also performed, to evaluate stent position and potential complications such as migration, granulation tissue formation at the edges of the stent, stent fracture and secretion plugs.

In order to objectively evaluate the amount of secretions accumulated in the stent area over time, we introduced in our clinical practice a 4-point endoscopic score, designed to quantify the amount of secretions during bronchoscopy at each follow-up visit. The score points are the following:

$0=$ no secretions;

$1=$ moderate amount of secretions easily removable by suction;

$2=$ severe amount of secretions removable using a biopsy forceps, instillation of mucolytic agents or other devices in addition to the suction;

3 = complete stent obstruction or deposit of thick and nonremovable secretions.

Institutional Review Board approval was obtained for this retrospective study.

\section{Statistical Analysis}

Data are expressed as means \pm standard deviation. Absolute values in each outcome variable were compared by Student's t test. Wilcoxon and Friedman tests were applied for non-parametric variables. A $p$ value of less than 0.05 was considered to indicate 
Table 1. Baseline characteristics of enrolled patients $(n=52)$

\begin{tabular}{lc}
\hline Age, years & $64 \pm 11$ \\
Male gender & $37(71)$ \\
BMI, kg/m ${ }^{2}$ & $25 \pm 4$ \\
Oxygen saturation, \% & $95 \pm 2.8$ \\
Comorbidities & $2.2 \pm 1.7$ \\
Stage & \\
$\quad$ IIIA & $5(10)$ \\
B & $21(42)$ \\
IV & $25(48)$ \\
ECOG score & $1.7 \pm 0.6$ \\
ASA score & $3 \pm 0.5$ \\
BI & $75 \pm 21$ \\
Etiology & $44(88)$ \\
Lung cancer & $18(40)$ \\
NSLC & $19(43)$ \\
NSCLC (squamous) & $7(15)$ \\
SCLC & $5(10)$ \\
Metastasis & $1(2)$ \\
Hemangiopericytoma & \\
Type of stenosis & $10(20)$ \\
Intrinsic obstruction & $12(24)$ \\
Extrinsic obstruction & $27(54)$ \\
Complex & $1(2)$ \\
Broncho-oesophageal fistula & \\
Follow-up, days & $22-549$ \\
Mean & 74 \\
Range & \\
Median & \\
\hline Values are presented as means \pm SD or numbers with percent- \\
ages in parentheses. BMI = Body mass \\
Cooperative Oncology Group; ASA American Society of Anes- \\
thesiologists; BI = bronchus intermedius; NSLC = non-small-cell \\
lung cancer; SCLC = small-cell lung cancer. \\
\hline
\end{tabular}

statistically significant differences. All analyses were performed using Statview software ${ }^{\circledR}$.

Patients with life expectancy less than 20 days were excluded from the statistical analysis.

\section{Results}

Stents were implanted in 52 patients, 14 females (mean age $64 \pm 10$ years, range $38-86$ years $)$, with malignant $(n=$ $48)$ and benign $(n=2)$ tracheobronchial strictures and malignant broncho-oesophageal fistula $(n=1)$. The baseline clinical characteristics are summarized in table 1.

All patients were symptomatic; the majority of them complained of dyspnoea $(n=29 ; 59 \%)$ of moderate degree (modified MRC $2.7 \pm 0.8)$ and cough $(n=25 ; 51 \%)$.

The main cause of airway obstruction was primary lung cancer ( 7 small-cell lung cancer, 20 squamous non-
Table 2. Characteristic details of stents

\begin{tabular}{llllll}
\hline Site & \multicolumn{2}{l}{ Type } & & & \multicolumn{2}{l}{ Measures, $\mathrm{mm}$} \\
\cline { 2 - 3 } \cline { 5 - 6 } & conical & linear & & length & diameter \\
\hline RMB & 3 & 2 & & $37.5 \pm 12.5$ & $12 \pm 1.6$ \\
LMB & 3 & 5 & & $35 \pm 7.5$ & $10.7 \pm 1.4$ \\
BI & 5 & 2 & & $32.8 \pm 9.5$ & $11.1 \pm 1.4$ \\
LMB + LLL & 1 & & & 40 & 14 \\
LLL & 6 & 21 & & $23.6 \pm 7.8$ & $10 \pm 0.9$ \\
BI + RLL & & 1 & & 60 & 11 \\
RLL & 1 & & & 20 & 10 \\
\hline
\end{tabular}

$\mathrm{RMB}=$ Right main bronchus; $\mathrm{LMB}=$ left main bronchus; $\mathrm{BI}=$ bronchus intermedius; LLL = left lower lobe bronchus. Length and diameters are expressed in millimetres as means \pm SD.

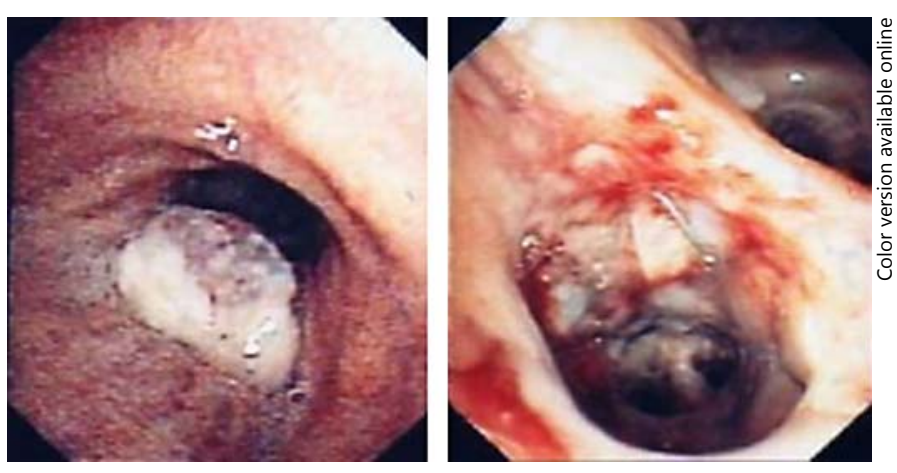

Fig. 1. Tumour of the left upper lobe which compresses the opening of the lower lobe bronchus. Silmet stent placed from the left main bronchus to the basal pyramid of the left lower bronchus.

small-cell lung cancer and 18 non-small-cell lung cancer). In 5 cases, the diagnosis was metastatic cancer (1 colon, 1 oesophageal, 1 endometrial and 2 laryngeal cancer) and in 1 case haemangiopericytoma. In 1 patient a conical stent was inserted into the right main bronchus to seal a broncho-oesophageal fistula. Two conical Silmet stents were placed into the trachea to treat a postintubation stenosis and a postradiotherapy malacia.

In 2 patients with malignant postobstructive abscess of the left lower lobe, stents were placed as a support drainage system with cleaning, sterilization and recovery of the residual lung.

Stents were inserted in the trachea $(n=19)$, in the main bronchi $(n=21)$ and in the peripheral bronchi $(n=31$; fig. 1). Details on stent characteristics and position are summarized in table 2. 


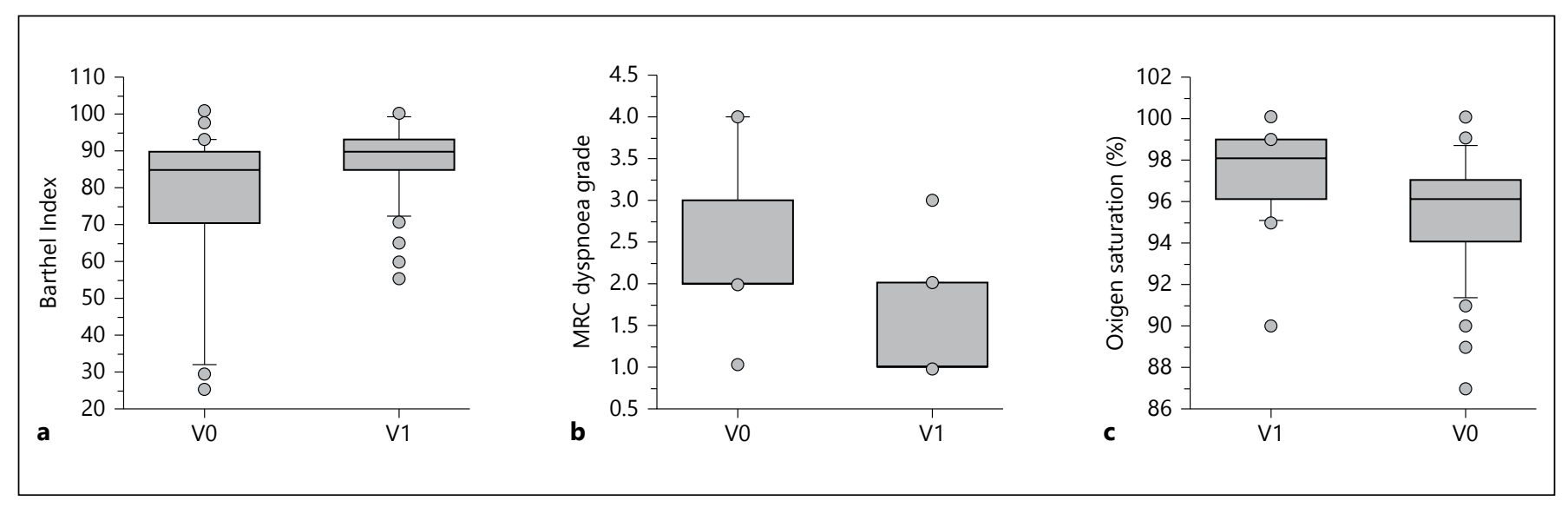

Fig. 2. Boxplot representation of difference before and after stent placement in: Barthel Index (a), MRC dyspnoea grade (b) and oxygen saturation (c).

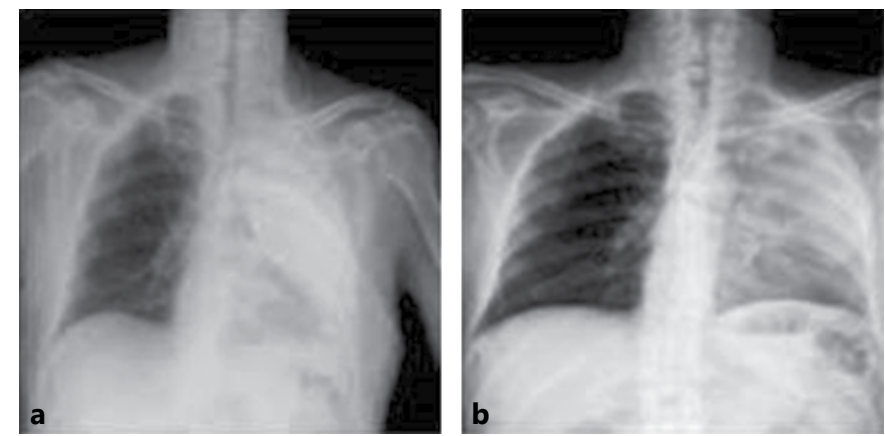

Fig. 3. Chest radiography performed before (a) and after (b) stenting showed maximal expansion and recovery of the residual lung.

It was not possible to perform lung function tests in all patients due to their poor clinical conditions.

Except for 1 dislocation, stent deployment was successful in all patients without procedure-related complications (such as death, stent rupture, severe bleeding, prolonged hypoxia, airway perforation, pneumothorax) and with immediate considerable symptomatic improvement, assessed by a statistically significant score difference in the Barthel Index (V0 Barthel Index median 69, range 25-93, and V1 Barthel Index median 90, range 35100; $\mathrm{p}<0.001$ ) and MRC score (V0 MRC median 3 and V1 MRC median 1; p < 0.001; fig. 2).

In $48 \%(25 / 52)$ of patients, a radiographic improvement was demonstrated by changing in computed tomography or chest radiography (fig. 3).

The mean duration of the procedure was $53 \pm 30 \mathrm{~min}$ (range $20 \pm 140$; median 47 ), and in $46 \%$ of cases laserassisted mechanical dilatation was used.
Early complication $(<24 \mathrm{~h})$ was recorded in 6 patients (7.6\%). Three patients needed mechanical ventilation in the postoperative period for less than $8 \mathrm{~h}$, and 2 experienced atrial fibrillation treated with pharmacological cardioversion with success.

We observed only 1 major complication during the stenting procedure: dislocation of the prosthesis in the subsegmentary bronchus of the right lower lobe above the stenosis, due to a technical error.

The mean follow-up duration was $119 \pm 120$ days (range 22-549 days; median 74 days).

Twenty-three patients (44\%) could be evaluated up to the 90th postoperative day, with a drop-out of $66 \% \mathrm{sec}-$ ondary to the fatal evolution of their malignant disease.

The overall complication rate of stent placement was $40 \%$. Types and timing of complications are summarized in table 3 .

Stents were removed from 7 patients $(15 \% ; n=3$ regression of tumour; $\mathrm{n}=3$ progression of tumour; $\mathrm{n}=1$ thermotherapy), after an average of $190 \pm 66$ days (range 55-430 days) without major complications, significant bleeding or stent rupture. A stent was replaced after a disobstruction procedure in the same section $(\mathrm{n}=1)$ and subsequently in case of recurrence $(n=1)$.

One preventive bronchial stent migrated after chemotherapy due to a significant reduction in tumour volume (fig. 4), and it was replaced with a larger Silmet stent because of residual bronchomalacia, with good results (fig. 5).

Two patients developed a small granuloma (3.8\%) that did not require laser treatment.

On average, the endoscopic secretion score was 0.7 at the first follow-up visit (V1), 1.1 at V2 and 1.5 at V3, with 
Table 3. Complication rates and time to detect complications after stent placement

\begin{tabular}{lll}
\hline Complication rate & $\begin{array}{l}\text { Patients, } \\
\mathrm{n}\end{array}$ & $\begin{array}{l}\text { Time after } \\
\text { implantation, } \\
\text { days }\end{array}$ \\
\hline $\begin{array}{lll}\text { Stent migration } \\
\quad \text { Postchemotherapy regression }\end{array}$ & $7(13.4)$ & $38 \pm 58$ \\
$\quad 3(5.7)$ & $45 \pm 120$ \\
$\quad$ of tumour & $1(1.9)$ & \\
$\quad$ Intraprocedural dislocation & $1(1.9)$ & 1 \\
$\quad$ Sislocation into cavity abscess & $2(3.8)$ & 30 \\
Granulation tissue formation & $2(3.8)$ & 30 \\
Infections & $3(5.7)$ & $30 \pm 60$ \\
Obstruction due to tumour outgrowth & $8(15)$ & $40 \pm 30$ \\
\hline
\end{tabular}

Results are presented as numbers with percentages in parentheses or means \pm SD.

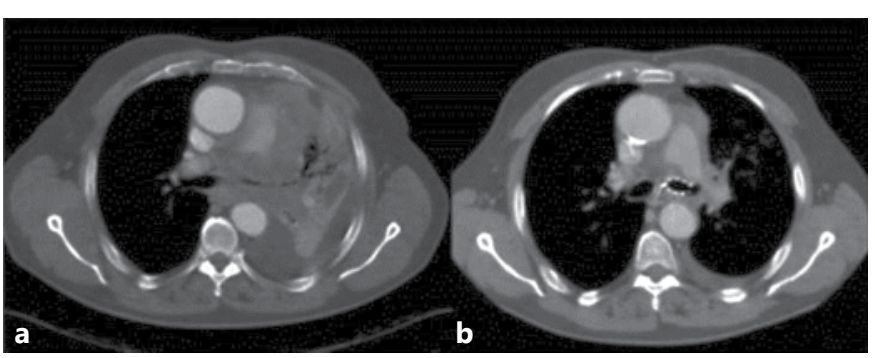

Fig. 4. Significant reduction in tumour volume at CT scan before (a) and after (b) chemotherapy.

a statistically significant difference between the visits (tied $\mathrm{p}$ value $<0.02$ ).

Two stent obstructions (3.8\%) were found due to tenacious secretions and were easily resolved with endoscopic toilette.

Culture of broncho-aspirates was performed in 30 patients (57\%); microbiological cultures were positive in 17 out of 30 patients ( $23 \%$ at 1 day, $58 \%$ at 30 days and $15 \%$ at 90 days). The pathogens isolated on bronchoaspirates were: Pseudomonas $(\mathrm{n}=2)$, Staphylococcus $(\mathrm{n}=6)$, Candida $(\mathrm{n}=5)$, Klebsiella $(\mathrm{n}=1)$, Sphingomonas paucimobilis $(\mathrm{n}=1)$, Enterobacteriaceae $(\mathrm{n}=2)$, Haemophilus $(\mathrm{n}=1)$, Streptococcus $(\mathrm{n}=1)$, Stenotrophomonas malthopilia $(\mathrm{n}=1)$, Comamonas testosteroni $(\mathrm{n}=1)$.

Clinical or radiological signs of infection were present in $9 / 17$ (52\%) of them and required targeted antibiotic therapy.

Completely Covered Self-Expandable Metal Stent in Tracheobronchial Disorders

\section{Discussion}

Tracheobronchial stenting is an appealing mini-invasive palliative treatment option for patients with tracheobronchial disorders, who are not surgical candidates.

Many different types of materials have been used to create a tracheobronchial prosthesis, and there are advantages and disadvantages for each of them.

Currently silicone stents are still the 'gold standard' for tracheobronchial disorders, as they are relatively easy to readjust, remove and replace.

To date, very few reports are available in the scientific literature about performance and clinical outcome of Silmet stents [24, 25].

In one study, Silmet stents were used to treat $21 \mathrm{pa}$ tients with tracheobronchial malignant stenosis, bronchopleural fistulae and oesotracheal fistula without complications except for a pneumothorax during stent deployment. In 2 case series, conical Silmet has proved to be an effective, safe and fast strategy to treat large postpneumonectomy bronchopleural fistula $[26,27]$.

This study represents a single referral institution experience in the management of malignant and benign tracheobronchial disorders with Silmet stents.

In our case series, the Silmet stent has been shown to be effective in relieving symptoms and improving quality of life, indeed with an objective radiographic amelioration. Stent placement was well tolerated with almost $60 \%$ of the patients experiencing no significant complication after a mean follow-up of 3.9 months.

We observed only 1 major stent-related complication with dislocation of the prosthesis during deployment in the subsegmentary bronchus of the right lower lobe, above the stenosis with no possibility of recovery; the patient was still alive at the 4th month of follow-up and had performed the first few cycles of chemotherapy with no stent-related complications recorded.

The rate of stent migration at 30 days not related to tumour regression was $7.6 \%$, and it was similar to data reported for the other SEMS such as the Ultraflex ${ }^{\mathrm{TM}}(4.7-$ $7.6 \%)[4,8,9,14,28,29]$ and Wallsten stent $(12 \%)[8,30]$.

In our series, none of the patients had tumour ingrowth, while it is reported as a quite common complication with the Ultraflex (5-21\%) and Wallsten stents (10\%). This could be probably related to the better biocompatibility of the Silmet stent, due to the mesh silicone cover and polyester external layer down to the edge of the device, compared to the other covered SEMS that have both their tips uncovered. 
Fig. 5. Loss of the stent's contact due to tumour shrinkage; it was replaced with a larger Silmet stent with good results.

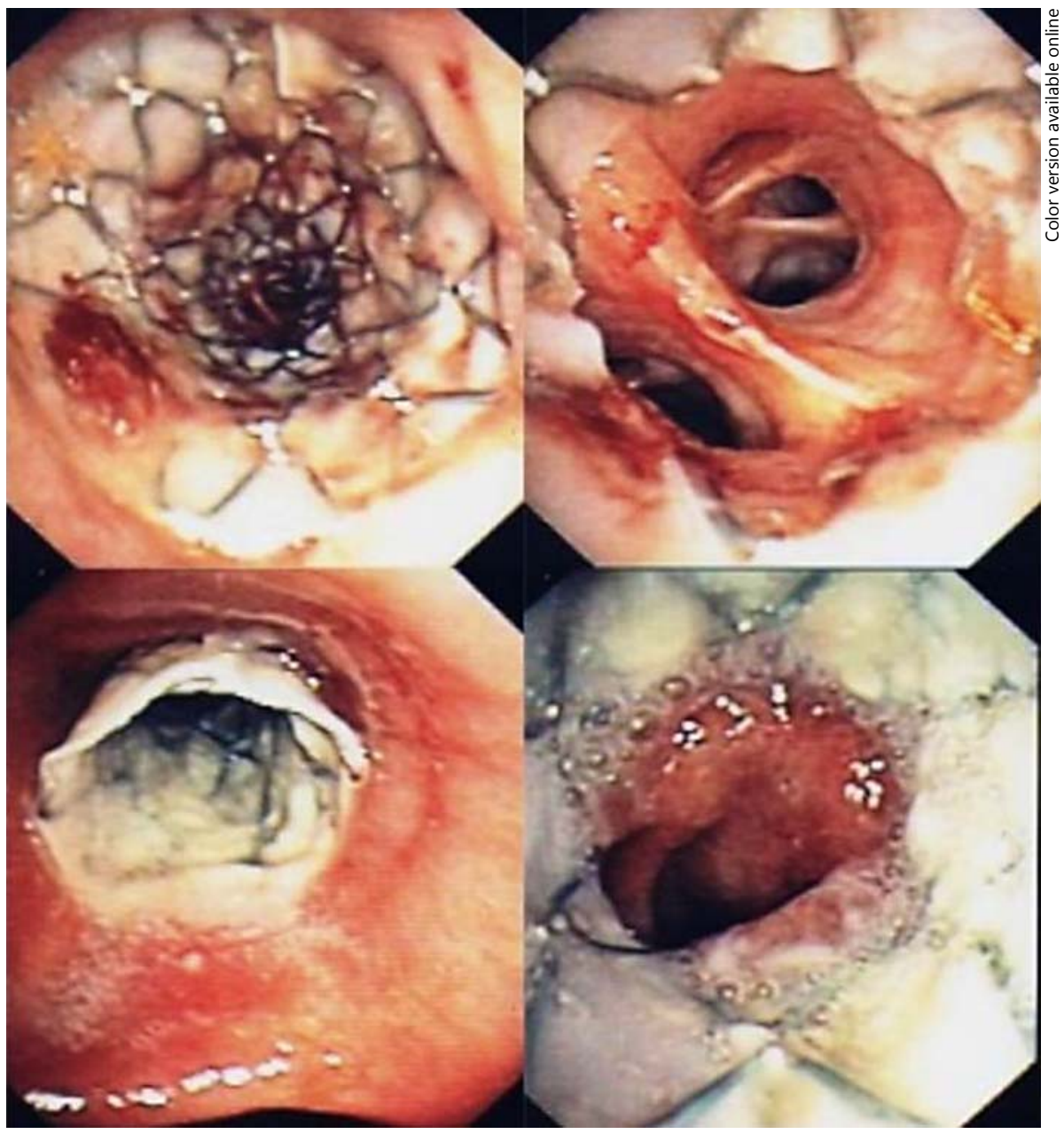

Overall, granuloma incidence (3.8\%) was within the low limit of range previously reported in the literature (2.9$15.2 \%)[4,8,9,14,28,30]$ with no need of laser treatment.

A possible explanation for such a low rate is that $92 \%$ of our patients underwent postprocedural treatment such as chemo- and radiotherapy. The immunosuppressive effect of these therapies could have reduced the inflammatory response and as a consequence, the tissue granulation formation, too.

It is well known that stents impair mucus clearance, thus accumulation of secretions can be considered a slightly annoying problem or a severe complication.

In order to explore stent performance in secretion retention, we introduced an endoscopic score of 4 points to quantify the amount of accumulated secretions at different visits. We believe this score is less operator dependent, because it is based on the method used to clean the stent rather than on the endoscopic appearance of accumulated secretions.
Obstruction due to tenacious secretions occurred in $3.8 \%$ of cases, less than reported in the literature for other SEMS (9-38\%) [4, 9, 30]; interestingly, an increase in the amount of secretions, measured by the endoscopic score, was observed from visit 0 to visit 1 but not during the following assessments, therefore we can state that the stent allowed a good drainage of secretions.

Despite the fact that the average follow-up time was not very long, we can assert that the Silmet stent has good mechanical properties. In fact, we did not observe any structural complications or failure in our series, not even in the 6 patients with longer survival or in the 6 patients in whom damage could have occurred, because of manipulation with strong bronchoscopic forceps during the removal.

Silmet stents have also been shown to be very versatile; in fact, in our series, approximately $50 \%$ of stents were placed in the peripheral bronchi, in the left and in the right lower lobe bronchus. Certainly, central airway ob- 
Table 4. Differences between patients depending on the site of obstruction

\begin{tabular}{lcc}
\hline Variables & \multicolumn{2}{l}{ Site } \\
\cline { 2 - 3 } & MB & LB \\
\hline Age, years & $63 \pm 11$ & $65 \pm 10$ \\
ECOG score & $1.8 \pm 0.5$ & $1.6 \pm 0.7$ \\
ASA score & $2.6 \pm 0.5$ & $3.1 \pm 0.5$ \\
Symptoms & & \\
$\quad$ Dyspnoea & $9(37.5)$ & $11(39.3)$ \\
$\quad$ Cough & $12(50)$ & $14(50)$ \\
$\quad$ Hemoptysis & $16(66.6)$ & $13(46.4)$ \\
Chest pain & $3(12.5)$ & $3(10.7)$ \\
MRC dyspnoea grade & & \\
$\quad$ V0 & $2.8 \pm 0.8$ & $2.5 \pm 0.9$ \\
$\quad$ V1 & $1.5 \pm 0.5$ & $1.3 \pm 0.7$ \\
Oxygen saturation, \% & & \\
$\quad$ V0 & $95.5 \pm 1.7$ & $95 \pm 3.6$ \\
$\quad$ V1 & $96.5 \pm 1.5$ & $97 \pm 3.6$ \\
Radiographic change, \% & 45 & 50 \\
Dislocation & $3(13)$ & $4(14)$ \\
Granulation tissue formation & 0 & $2(7.4)$ \\
Infections & $2(8.6)$ & $1(3.7)$ \\
Obstruction due to tumour outgrowth & $3(13)$ & $5(18)$ \\
\hline
\end{tabular}

Values are presented as means \pm SD or numbers with percentages in parentheses. $\mathrm{MB}=$ Main bronchi; $\mathrm{LB}=$ lobar bronchi; ECOG $=$ Eastern Cooperative Oncology Group; ASA = American Society of Anesthesiologists.

structions are life-threatening conditions that lead to severe respiratory symptoms but from a technical and procedural point of view, their stenting is less complicated compared to distal airways. Moreover, very often patients present with locally advanced disease involving lobar bronchi and the secondary carina, right at the time of diagnosis or during the follow-up. Furthermore, although patients with distal airway obstruction are paucisymptomatic, mainly complaining of chronic cough, mild dyspnoea or recurrent infections, they are at high risk of disease progression and complication, such as recurrent postobstructive pneumonia, that may seriously interfere with the administration of additional medical treatment (chemotherapy or radiotherapy) and may worsen their performance status.

Previously, Breitenbücher et al. [9] - in their study on the management of complex malignant airway stenosis - placed Ultraflex stents in the lobar bronchi of 7 patients. They reported that survival after distal stent placement was longer than after proximal stenting, possibly because the patients had a smaller tumour load. Moreover, they recommend stent placement in distal airways only in cases of extrinsic compression without endoluminal growth, because the shorter Ultraflex stent, needed for these sites on the tracheobronchial tree, are uncovered [28].

We used the Silmet stent not only for the management of stenoses due to extrinsic compression ( $\mathrm{n}=12 ; 24 \%)$, but also due to intrinsic $(\mathrm{n}=10 ; 20 \%)$ and complex obstruction $(\mathrm{n}=27 ; 54 \%)$. In fact the fully covered Silmet stents are available in lengths from 20 to $60 \mathrm{~mm}$ (in increments of $10 \mathrm{~mm}$ ).

Moreover, in 2 patients the Silmet stent was favourably used to drain a postobstructive abscess of the left lower lobe obtaining negative microbiological cultures and the absence of respiratory symptoms at the second month of follow-up.

Furthermore, in our series 2 patients with benign airway strictures (a posttracheostomy stenosis and a postradiotherapy malacia) were successfully treated with a Silmet stent, achieving a good palliation, without major complications during the 146.4 days of follow-up; we understand that it is not possible to draw any conclusion with such an exiguous number of cases, but it may be a starting point for future research.

In summary, our study emphasizes that the Silmet stent is a feasible, effective and safe palliative treatment for neoplastic and benign tracheobronchial strictures.

To our knowledge, this is the first report describing the use of the Silmet stent in the treatment of small- to medium-caliber airways.

\section{Conclusion}

The silicone stent remains the gold standard for the treatment of airway strictures. However, stent type should be chosen depending on the lesion's characteristics and airway anatomy which can be evaluated only during intra-operative procedures.

We can conclude that the Silmet stent is safe and simple to implant and remove. We suggest its use in cases of tight stenosis, in the treatment of small- to medium-caliber airways or in cases of tortuous airways. We can also hypothesize their utility in the treatment of benign stenosis, since we had a favourable experience, but a larger number of cases is necessary to prove it.

Further randomized controlled trials comparing patients treated with different types of stent are needed to assess the overall advantages of using Silmet versus other types of prosthesis. 


\section{References}

$\checkmark 1$ Freitag L: Tracheobronchial stents; in Bolliger 11 Vergnon JM, Costes F, Bayon MC, Emonot A: CT, Mathur PN (eds): Interventional Bronchoscopy. Basel, Karger, 2000, pp 171-186.

2 Gompelmann D, Eberhardt R, Herth FJF: Advanced malignant lung disease: what the specialist can offer. Respiration 2011;82:111-123.

-3 Wood DE, Liu YH, Vallières E, Karmy-Jones R, Mulligan MS: Airway stenting for malignant and benign tracheobronchial stenosis. Ann Thorac Surg 2003;76:167-172.

4 Mroz RM, Kordecki K, Kozlowski MD, et al: Severe respiratory distress caused by central airway obstruction treated with self-expandable metallic stents. J Physiol Pharmacol 2008; 59(suppl 6):491-497.

5 Miyazawa T, Miyazu Y, Iwamoto Y, et al: Stenting at the flow-limiting segment in tracheobronchial stenosis due to lung cancer. Am J Respir Crit Care Med 2004;169:10961102.

-6 Tanigawa N, Sawada S, Okuda Y, Kobayashi M, Mishima K: Symptomatic improvement in dyspnea following tracheobronchial metallic stenting for malignant airway obstruction. Acta Radiol 2000;41:425-428.

7 Rieger J, Hautmann H, Linsenmaier U, et al: Treatment of benign and malignant tracheobronchial obstruction with metal wire stents: experience with a balloon-expandable and a self-expandable stent type. Cardiovasc Intervent Radiol 2004;27:339-343.

-8 Saad CP, Murthy S, Krizmanich G, Mehta AC: Self-expandable metallic airway stents and flexible bronchoscopy: long-term outcomes analysis. Chest 2003;124:1993-1999.

-9 Breitenbücher A, Chhajed PN, Brutsche MH, Mordasini C, Schilter D, Tamm M: Longterm follow-up and survival after Ultraflex stent insertion in the management of complex malignant airway stenoses. Respiration 2008; 75:443-449.

10 Fournel C, Bertoletti L, Nguyen B, Vergnon $\mathrm{J}-\mathrm{M}$ : Endobronchial metastases from colorectal cancers: natural history and role of interventional bronchoscopy. Respiration 2009; 77:63-69. Efficacy of tracheal and bronchial stent placement on respiratory functional tests. Chest 1995;107:741-746.

12 Bolliger CT, Breitenbuecher A, Brutsche M, Heitz M, Stanzel F: Use of studded Polyflex stents in patients with neoplastic obstructions of the central airways. Respiration 2004;71: 83-87.

13 Amjadi K, Voduc N, Cruysberghs Y, Lemmens R, Fergusson DA, Doucette S, Noppen MP: Impact of interventional bronchoscopy on quality of life in malignant airway obstruction. Respiration 2008;76:421-428.

14 Murgu S, Langer S, Colt H: Bronchoscopic intervention obviates the need for continued mechanical ventilation in patients with airway obstruction and respiratory failure from inoperable non-small-cell lung cancer. Respi-

15 Razi SS, Lebovics RS, Schwartz G, Sancheti M, Belsley S, Connery CP, Bhora FY: Timely airway stenting improves survival in patients with malignant central airway obstruction. Ann Thorac Surg 2010;90:1088-1093.

16 Prashant N: Therapeutic bronchoscopy for malignant airway stenosis: choice of modality and survival. J Cancer Res Ther 2010;6:204209.

17 Ernst A: Stenting of the tracheobronchial tree; in Simoff MJ, Sterman DH, Ernst A (eds): Thoracic Endoscopy: Advances in Interventional Pulmonology. Oxford, Blackwell Publishing, 2006, pp 167-172.

18 Dasgupta A, Dolmatch BL, Abi-Saleh WJ, Mathur PN, Mehta AC: Self-expandable metallic airway stent insertion employing flexible bronchoscopy. Preliminary results. Chest 1998;114:106-109.

19 Dooms C: Performance of fully covered selfexpanding metallic stents in benign airway strictures. Respiration 2009;77:420-426.

20 Miyazawa T, Yamakido M, Ikeda S, et al: Implantation of Ultraflex nitinol stents in malignant tracheobronchial stenosis. Chest 2000; 118:959-965.

21 Sparup J, Borgeskov S: Hjertecentret. Self-expanding nitinol stents in the treatment of tracheobronchial stenoses. Ugeskr Laeger 2002; 164:3858-3861. ration 2012;84:55-61.
22 Shin J, Song H, Ko G, et al: Treatment of tracheobronchial obstruction with a polytetrafluoroethylene covered retrievable expandable nitinol stent. J Vasc Interv Radiol 2006; 17:657-663.

23 Trisolini R, Paioli D, Fornario V, et al: Collapse of a new type of self-expanding metallic tracheal stent. Monaldi Arch Chest Dis 2006; 65:56-58.

24 Creagh-Brown B, Sheth A, Crerar-Gilbert A, et al: A novel approach to the management of acute tracheal tear. J Laryngol Otol 2008;21: $1-2$.

25 Duysinx B, Louis R, Corhay JL: Early experience of a new nitinol expandable stent in tracheobronchial diseases. Oral presentation ERS, Munich, 2-6 September 2006. Eur Respir J 2006;28(suppl 50):371s.

26 Dutau H, Breen DP, Gomez C, Thomas PA, Vergnon JM: The integrated place of tracheobronchial stents in the multidisciplinary management of large post-pneumonectomy fistulas: our experience using a novel customised conical self-expandable metallic stent. Eur J Cardiothorac Surg 2011;39:185-189.

27 Chae EY, Shin JH, Song HY, Kim JH, Shim TS, Kim DK: Bronchopleural fistula treated with a silicone-covered bronchial occlusion stent. Ann Thorac Surg 2010;89:293-296.

28 Bollinger CT: The use of the covered Wallstent for the palliative treatment of inoperable tracheobronchial cancers. Chest 1996;110: 1161-1168.

29 Chung FT, Lin SM, Chen HC, Chou CL, Yu CT, Liu CY, Wang $\mathrm{CH}$, Lin HC, Huang CD, Kuo HP: Factors leading to tracheobronchial selfexpandable metallic stent fracture. J Thorac Cardiovasc Surg 2008;136:1328-1335.

30 McGrath EE, Warriner D, Anderson P: The insertion of self-expanding metal stents with flexible bronchoscopy under sedation for malignant tracheobronchial stenosis: a singlecenter retrospective analysis. Arch Bronchopneumol 2011;48:43-48. 